\title{
Auf dem Weg zu einer strategischen Partnerschaft: Die Stärkung der OSZE durch eine bessere Zusammenarbeit mit der EU
}

\author{
David Galbreath, André Härtel, Stefan Wolff
}

\section{Zusammenfassung}

Die EU und die OSZE stehen ganz unterschiedlichen politischen und strukturellen Herausforderungen gegenüber. Der neue strategische Kompass der EU betont ihren Anspruch, ein ernstzunehmender Sicherheitsakteur zu werden. Die OSZE muss mit einem schwindenden Wertekonsens sowie geschwächten Durchführungsorganen und Institutionen umgehen. Kann die OSZE durch eine verbesserte Zusammenarbeit mit der EU wiederbelebt werden - und wenn ja, wie kann dies gelingen? Die Autoren erläutern, warum der Aufstieg der EU als Sicherheitsakteur nicht auf Kosten der OSZE gehen sollte. Die Zusammenarbeit zwischen den beiden Organisationen sollte sich auf folgende Punkte konzentrieren: (1) eine Stärkung der OSZE als autonome Sicherheitsorganisation, (2) die Nutzung der OSZE als ein Forum für Dialog und den Aufbau von Vertrauen sowie (3) die gewinnbringende Kombination der wesentlichen Stärken beider Organisationen und die Vermeidung von Dopplungen im operativen Handeln.

\section{Schlagworte}

OSZE, EU, europäische Sicherheit, strategische Partnerschaft, interorganisationale Zusammenarbeit

Bitte zitieren als: David Galbreath, André Härtel und Stefan Wolff: Auf dem Weg zu einer strategischeren Partnerschaft: Die Stärkung der OSZE durch eine bessere Zusammenarbeit mit der EU, OSCE Insights 3/2021 (Baden-Baden: Nomos, 2022), https://doi.org/10.5771/978374891 1449-03

\section{Einleitung}

Die EU und die OSZE befinden sich in einer entscheidenden Phase: Es geht um ihre Rollen innerhalb der europäischen Sicherheitsordnung. Die EU will

* David Galbreath

Department of Politics, Languages and International Studies University of Bath dg341@bath.ac.uk im März 2022 einen „strategischen Kompass" verabschieden. Er soll die EU in die Lage versetzen, weltweiten Sicherheitsgefahren und -herausforderungen zu begeg-
André Härtel
Department for Political Science
Friedrich Schiller University Jena
andre.haertel@gmail.com
Stefan Wolff
Department of Political Science and Interna- tional Studies 
nen. ${ }^{1}$ Das Ziel lautet dabei, „eine gemeinsame europäische Sicherheits- und Verteidigungskultur zu stärken“ und „die passenden Absichten und konkreten Ziele [zukünftiger] politischer Strategien zu definieren “.2 Laut dem European Union Institute for Security Studies soll der strategische Kompass „eine politische Orientierung für die Sicherheit und Verteidigung der EU bieten sowie die operative Effektivität, die Widerstandsfähigkeit und das Leistungsvermögen der Union ebenso wie ihre Zusammenarbeit mit Partnern verbessern". 3

Die OSZE steht anderen Herausforderungen gegenüber, wie den zunehmenden internen Spannungen und der Tatsache, dass sie ihrem umfassenden Sicherheitsmandat immer weniger gerecht werden kann. ${ }^{4}$ Aufgrund der internen Spannungen erodiert der Konsens über die Normen, auf denen die OSZE seit den 1970er-Jahren beruht. Das schwächt zugleich die Durchführungsorgane und Institutionen der OSZE. 5 Dadurch wird der Organisation eine geringere politische Bedeutung zugeschrieben und die Teilnehmerstaaten sind weniger gewillt, politische und finanzielle Mittel für die OSZE bereitzustellen. Wolfgang Zellner führt an, dass die OSZE „als inklusive Plattform und Akteur in Situationen, in denen andere IOs [internationale Organisationen] nicht handlungsfähig sind, "6 zwar eindeutig benötigt werde - doch innerhalb der europäischen Sicherheitsord-

University of Birmingham

s.wolff@bham.ac.uk nung sei sie an den Rand gedrängt worden.

Der strategische Kompass der EU umfasst die vier „Körbe“ Krisenmanagement, Resilienz, Fähigkeiten und Partnerschaften. $^{7}$ Der Korb „Partnerschaften“ bietet der EU und der OSZE eine neue Gelegenheit, ihre strategische Partnerschaft zu überdenken. Die Zusammenarbeit zwischen den beiden Organisationen hat eine lange Geschichte. Die gemeinsamen Interessen und die Vorteile einer Zusammenarbeit wurden von Vertreter*innen der OSZE und der EU immer wieder betont. ${ }^{8}$ In ihrer Globalen Strategie des Jahres 2016 erkannte die EU die OSZE formal „als europaweite Organisation, [die] sich im Zentrum der europäischen Sicherheitsordnung befindet" an. Sie verpflichtete sich, „ihren Beitrag innerhalb der OSZE und ihre Zusammenarbeit mit der OSZE als Säule der europäischen Sicherheit zu stärken“.9 Bei der Überprüfung ihrer Globalen Strategie im Jahr 2019 bekräftigte die EU ihre Unterstützung für die OSZE. ${ }^{10}$ Dieses gemeinsame Interesse an Sicherheit und Stabilität äuBerte sich auch in den Prioritäten der EU für die östliche Partnerschaft wie gute Regierungsführung, Kampf gegen die organisierte Kriminalität, Bekämpfung von Korruption und der Umgang mit Sicherheitsherausforderungen, die der Klimawandel mit sich bringt. ${ }^{11}$

Die Wirklichkeit sieht jedoch anders aus. Die beiden Organisationen verfolgen keinen gemeinsamen Ansatz bei Sicherheitsfragen und arbeiten eher parallel, anstatt an einem Strang zu ziehen. ${ }^{12}$ Trotz positiver Gegenbeispiele - wie etwa die Zusammenarbeit zwischen der aktuellen 
OSZE-Sonderbeobachtermission in der Ukraine und der dortigen European Union Advisory Mission - hat sich diese Tendenz in letzter Zeit noch verstärkt. Die Spannungen innerhalb der OSZE sind weiter gewachsen, und die EU bemüht sich mittlerweile um eine größere Autonomie als Sicherheitsakteur in Europa, vor allem seit dem Vertrag von Lissabon aus dem Jahr 2007.

Eine fähigere und selbstbewusstere EU kann (und sollte) zwar mehr zur europäischen Sicherheit beitragen, aber wir sind der Auffassung, dass dies nicht auf Kosten der OSZE geschehen sollte. Im Gegenteil. Nicht nur ist "das Überleben der OSZE objektiv im Interesse der EU“. ${ }^{13}$ Eine gestärkte OSZE würde auch den nationalen Interessen der 27 EU-Mitgliedstaaten und der 57 OSZE-Teilnehmerstaaten am meisten dienen. ${ }^{14}$

\section{Die Zusammenarbeit zwischen der EU und der OSZE}

Beide Organisationen haben erst Anfang der 2000er-Jahre damit begonnen, ihre Zusammenarbeit zu konkretisieren, obwohl sich die Europäische Kommission und die EU bereits seit 1975 auf die KSZE-/OSZE-Prinzipien verpflichtet hatten (durch die Unterzeichnung der Schlussakte von Helsinki 1975, der Charta von Paris 1990 sowie der Europäischen Sicherheitscharta 1999). Im Jahr 1999 rief die OSZE ihre „Plattform für kooperative Sicherheit" ${ }^{15}$ ins Leben und die Europäische Kommission veröffentlichte 2003 ihre Schlussfolgerungen zur „EU-OSZEKooperation bei der Konfliktverhütung, dem Krisenmanagement und der Konfliktnachsorge“. ${ }^{16}$ Darin wurde die Notwendigkeit betont, „Doppelarbeit zu vermeiden" und auf eine "effektive gegenseitige Ergänzung“ hinzuarbeiten. Insbesondere Erkundungsmissionen, die Koordination vor Ort sowie gegenseitige diplomatische Unterstützung wurden als Prioritäten bei der Zusammenarbeit genannt. ${ }^{17}$

Seit 2006 übernimmt die EU eine formale Rolle innerhalb der OSZE: „Bei Sitzungen der Beschlussfassungsorgane sitzt die Europäische Kommission neben dem Teilnehmerstaat, der zu diesem Zeitpunkt den EU-Vorsitz innehat.“ Sie kann „unmittelbar nach dem Teilnehmerstaat, der den EU-Vorsitz innehat, das Wort ergreifen." ${ }^{18}$ Diese Rolle wurde 2018 auf Dienststellenebene in einem Briefwechsel zwischen den Generalsekretären der OSZE, der Europäischen Kommission und des Europäischen Auswärtigen Dienstes weiter formalisiert. Darin verpflichteten sich beide Organisationen zu regelmäßigen Beratungen und zu einer operativen Zusammenarbeit in Bereichen von gemeinsamem Interesse in allen drei OSZE-Dimensionen (politisch-militärische Dimension, Wirtschaftund Umweltdimension sowie menschliche Dimension). ${ }^{19}$ Zudem nimmt der Hohe Vertreter der EU für Außen- und Sicherheitspolitik und Vizepräsident der EU normalerweise auch an dem jährlichen OSZE-Ministerratstreffen im Dezember teil.

Die Arbeit der EU innerhalb der OSZE wird von der entsprechenden EUDelegation bei internationalen Organisationen in Wien geleitet. Sieben Mit- 
arbeiter*innen widmen sich ausschließlich einzelnen OSZE-Institutionen oder themenspezifischen Dimensionen. ${ }^{20} \mathrm{Da}$ rüber hinaus verfügt die Europäische Kommission über eine Arbeitsgruppe "OSZE und Europarat", die sich mit den Beziehungen der EU zu diesen beiden Organisationen beschäftigt und die gemeinsamen Positionen der Mitgliedstaaten in den Debatten in OSZE und Europarat koordiniert. ${ }^{21}$

Den Teilnehmerstaaten der OSZE ist es nicht gelungen, sich auf die Gründung eines Verbindungsbüros in Brüssel zu verständigen. In Wien sind die Abteilung für externe Zusammenarbeit des Büros des Generalsekretärs und ein Leitender Beauftragter für externe Zusammenarbeit (für alle Institutionen in Brüssel) für die Beziehungen zwischen der OSZE und der EU zuständig. Dass die OSZE über keine direkteren Verbindungsstrukturen verfügt - vor allem nicht in den Feldmissionen -, hat die systematische Koordinierung von Aktivitäten mit Brüssel erschwert.

Bis vor Kurzem haben beide Organisationen das Format ihrer Kooperation flexibel gehandhabt, und zwar durch unregelmäßige Treffen zwischen der EU und den OSZE-Troikas, Treffen zwischen dem Generalsekretär der OSZE und dem Hohen Vertreter der EU für Außenund Sicherheitspolitik und jährlich stattfindende Meetings auf Mitarbeiterebene. In einem Briefwechsel des Jahres 2018 wurden Bereiche mit gemeinsamen Interessen identifiziert, in denen sich das gesamte Spektrum an OSZE-Aktivitäten widerspiegelt. Dazu gehören Konfliktverhütung und Vermittlungstätigkeiten, die
Bekämpfung des Terrorismus sowie die Förderung von Rechtsstaatlichkeit und guter Regierungsführung. Diese große Bandbreite macht es schwierig, eine Agenda festzulegen und strategische Prioritäten zu setzen. Hinzu kommen die Fluktuation auf leitender Personalebene beider Seiten und strukturelle Unverträglichkeiten, die die Suche nach den richtigen Gesprächspartner*innen und die Etablierung effektiver Beziehungen zwischen ihnen behinderten.

\section{Gegenseitige Ergänzung}

Die Forschung zu interorganisationaler Zusammenarbeit zeigt, dass der Austausch von Ressourcen den wesentlichen Anreiz für eine Kooperation zwischen Organisationen darstellt. Internationale Organisationen kooperieren miteinander, weil sie an speziellen Mitteln (Gelder für Projekte, Infrastruktur, Personal) oder immateriellen Gütern (Legitimierung, Fachkenntnisse, Ruf) interessiert sind, die der jeweilige Partner zur Verfügung stellen kann. ${ }^{22}$ Der Austausch von Ressourcen beruht also auf einander ergänzenden Interessen zwischen den zusammenarbeitenden Organisationen. Auch einander überschneidende politische Strategien können ein wichtiger Anreiz für eine Kooperation und einen Ressourcenaustausch sein.

Die EU ist zu einer wichtigen Finanzierungsquelle der OSZE geworden - jedenfalls in den Bereichen, in denen sich die Interessen der EU mit dem Bedarf der OSZE decken. Die EU ist mit Abstand der größte Geldgeber für die OSZE-Son- 
derbeobachtermission in der Ukraine. Sie stellt Personal, Ausrüstung und Satellitenbilder zur Verfügung. ${ }^{23}$ Dank ihres Netzwerks aus Delegationen im ganzen OSZE-Gebiet kann die EU den OSZEFeldmissionen zudem eine maßgeschneiderte Unterstützung bieten.

Die OSZE wiederum verfügt über erhebliche (wenngleich zur Zeit geringer werdende) Möglichkeiten, Treffen einzuberufen und Agenden festzusetzen. Sie verfügt über Kenntnisse und Werkzeuge bei der Konfliktverhütung, Erfahrung in der stillen Diplomatie und ein echtes multilaterales Mandat im Zusammenhang mit Konflikten. Im Vergleich zur EU - die in den langwierigen Konflikten in der OSZE-Region nicht als neutraler Akteur wahrgenommen wird - ist es für die OSZE leichter, als Vermittler und/oder Beobachter vor Ort akzeptiert zu werden. Ungeachtet der aktuellen politischen Meinungsverschiedenheiten zwischen den OSZE-Teilnehmerstaaten ist die Organisation nach wie vor eines der wenigen europaübergreifenden Foren und deswegen für die EU sehr nützlich, etwa für einen institutionalisierten Austausch mit Russland über Sicherheits- und andere Fragen. Vor Ort verfügt die OSZE immer noch über wesentlich höhere Fachkenntnisse als die EU, beispielsweise bei der Konfliktvermittlung. Die OSZE-Feldmissionen können die EU unterstützen, etwa wenn es darum geht, die Standpunkte und den Bedarf von nationalen Minderheiten zu ermitteln. Darüber hinaus kann die OSZE als Bindeglied fungieren, etwa bei der Umsetzung der Zentralasienstrategie der EU.

\section{Hindernisse}

Abgesehen vom Ressourcenbedarf gibt es noch weitere zentrale Faktoren, die die Zusammenarbeit zwischen Organisationen beeinflussen: die Präferenzen der Mitgliedstaaten, das Machtungleichgewicht zwischen diesen bzw. der Organisation, die Kultur und Offenheit der Organisation, die Rolle des interorganisationalen Lernens bzw. frühere „Kooperationspfade" sowie der Einfluss mächtiger Drittparteien. ${ }^{24}$

$\mathrm{Zu}$ den Hindernissen für eine aktivere Partnerschaft zwischen EU und OSZE gehört das Ungleichgewicht hinsichtlich Macht, Budgets und struktureller Eigenschaften. Die EU ist nicht per se eine internationale Organisation, sondern ein Staatenverbund. Sie verfügt über einen Haushalt von etlichen Milliarden Euro und die einzelnen Institutionen sind durchaus unabhängig. Selbst in ihrer Außenpolitik weicht die Union immer häufiger vom Einstimmigkeitsprinzip ab. Am anderen Ende des Spektrums befindet sich die OSZE als eher fragmentierte Organisation mit einem umständlichen juristischen Status. Ihre Beschlussfassung erfordert einen Konsens, und der jährliche Haushalt beträgt knapp unter 140 Millionen Euro. ${ }^{25}$ Eine Konsequenz dieser asymmetrischen Beziehung ist die verständliche Sorge der OSZE (die viele einflussreiche Teilnehmerstaaten teilen), von der EU in eine Abhängigkeit gedrängt zu werden oder sich zu einem außen- und sicherheitspolitischen Werkzeug der EU zu entwickeln.

Weitere Unklarheiten bestehen bei Mitgliedschaft, Mandaten und Bestrebun- 
gen. Obwohl die OSZE alle 27 EU-Mitgliedstaaten umfasst, gehören zu ihren Teilnehmerstaaten auch Russland, weitere postsowjetische Staaten, die USA, Kanada, Norwegen und das Vereinigte Königreich. ${ }^{26}$ Aufgrund der höheren Bandbreite an Regimetypen und außenpolitischen Orientierungen innerhalb der OSZE fehlt ihr die Kohärenz der EU. Sie versteht sich eher als Forum denn als voll ausgebildete internationale Organisation. ${ }^{27}$ Für eine effektive Zusammenarbeit müssen die EU-Mitgliedstaaten deswegen zwischen ihren Präferenzen als EU-Mitglieder und als OSZE-Teilnehmerstaaten unterscheiden. Wenn die EU die OSZE nicht als eigenständige Sicherheitsorganisation behandelt, wird es für Russland und andere Teilnehmerstaaten, die nicht zur EU gehören, inakzeptabel sein, dass die OSZE enger mit der EU zusammenarbeitet und die EU somit innerhalb der OSZE an Sichtbarkeit und Einfluss gewinnt.

Die EU bemüht sich zunehmend und ernsthaft darum, eine Sicherheitsorganisation zu werden. Die OSZE hingegen hat Schwierigkeiten, in ihren Kernbereichen Konfliktverhütung und Vermittlung relevant zu bleiben. Sie arbeitet an Themen, bei denen alle Teilnehmerstaaten einer Meinung sind, die ihr Mandat aber eher am Rande betreffen und an anderer Stelle effektiver behandelt werden könnten. Dies wirft die Frage auf, inwiefern die EU die OSZE überhaupt benötigt, um ihre eigenen Interessen zu verfolgen, und ob die unausweichliche Doppelarbeit dazu beitragen könnte, dass die OSZE weiter marginalisiert wird.
Die Grundlage für eine erfolgreiche Zusammenarbeit zwischen internationalen Organisationen ist eine sensible Handhabung der Beziehung zueinander, vor allem angesichts sich überschneidender Mitgliedschaften. Zu diesem Zweck muss eine echte Vision entwickelt werden, wie diese Zusammenarbeit aussehen soll. Es muss regelmäßige Treffen geben, und die Art und Weise, wie interagiert wird, muss geplant werden. ${ }^{28}$ An dem Management der Beziehung zwischen der EU und der OSZE wird noch gearbeitet. Aktuell ist die Beziehung nicht hinreichend institutionalisiert, und selbst nach dem Briefwechsel von 2018 geben hochrangige Vertreter*innen zu, dass weitere Anstrengungen vonnöten sind.

Und in der Tat scheint es keinen klaren Plan zu geben, wie eine Zusammenarbeit aussehen könnte - etwa, dass die verschiedenen OSZE-Abteilungen an die Kapazitäten der Europäischen Kommission und ihre Finanzierungsmöglichkeiten für die Konfliktverhütung und das Konfliktmanagement angebunden werden. Außerdem ist die Liste konkreter Bereiche, in denen eine Zusammenarbeit stattfinden sollte, unzusammenhängend und zu lang. Es wäre deswegen sinnvoll, Prioritäten zu identifizieren und klare Ziele zu setzen.

Die alltägliche informelle Zusammenarbeit zwischen den beiden Organisationen wird oft gelobt. Allerdings gibt es dafür wenig Belege (im Gegensatz zu der regelmäßigen Doppelarbeit und den parallelen Vorgehensweisen, die in der entsprechenden Forschung dokumentiert sind). ${ }^{29}$ Helga Schmid, frühere Generalsekretärin des Europäischen Auswärtigen 
Dienstes und aktuelle Generalsekretärin der OSZE, könnte eine wichtige Rolle spielen: Als „Boundary Spanner“ könnte sie es beiden Organisationen ermöglichen, effektiver zusammenzuarbeiten.

\section{Drei Bausteine für eine bessere Partnerschaft}

Wir schlagen drei Bausteine vor, mit denen die Partnerschaft zwischen der OSZE und der EU verbessert werden kann. Der erste Baustein ist eine realistische Bewertung dessen, was eine intensivere Zusammenarbeit zwischen der EU und der OSZE erreichen kann. Die OSZE darf nicht bloß zu einem Instrument der Sicherheitsinteressen der EU werden. Sie muss gestärkt werden - als ein autonomer Sicherheitsakteur mit Fähigkeiten, die sich von denen der EU unterscheiden, für den Umgang mit gemeinsamen $\mathrm{Si}$ cherheitsherausforderungen aber relevant sind.

Der zweite Baustein: Die EU muss die OSZE als Dialogforum verstehen, nutzen und stärken. Nur so kann gegenseitiges Vertrauen aufgebaut werden. Dies erfordert eine größere Bereitschaft der EU, sich auf Akteure in Russland und Ländern „östlich der EU“ einzulassen, etwa wenn es um die Bedeutung und die Interpretation bestehender OSZE-Verpflichtungen und umstrittene Konzepte wie "Souveränität" und „Selbstbestimmung“ geht. Damit soll sichergestellt werden, dass sich die Streitigkeiten innerhalb der europäischen Sicherheitsordnung nicht verfestigen und eines Tages unüberwindbar werden. ${ }^{30}$
Der dritte Baustein: Eine Konzentration auf die gemeinsamen Interessen der 27 EU-Mitglieder bzw. der 57 OSZE-Staaten würde es ermöglichen, sich der Wurzeln der Zusammenarbeit zu besinnen, Doppelarbeit zu vermeiden und die größten Stärken beider Organisationen in allen drei OSZE-Dimensionen gewinnbringend einzusetzen.

\section{Empfehlungen für eine engere Zusammenarbeit}

Auf Grundlage dieser drei Bausteine für eine bessere Partnerschaft empfehlen wir der OSZE und ihren Teilnehmerstaaten sowie der EU und ihren Mitgliedstaaten sieben konkrete Schritte.

\section{a) Stärkung der Konfliktverhütung}

Die EU und die OSZE weisen unterschiedliche, aber teilweise deckungsgleiche Stärken bei der Konfliktverhütung auf. Die EU besitzt besser entwickelte Instrumente zur strukturellen Konfliktverhütung. Die OSZE kann Erfolge bei der direkten Prävention aufweisen und verfügt über die entsprechenden Fähigkeiten, aber ihr fehlen oft die finanziellen Ressourcen, um rasch zu handeln. Eine bessere Zusammenarbeit würde deswegen bedeuten, die Präventionsstrategien enger miteinander $z u$ verbinden und sie zu operationalisieren. Die OSZE könnte der EU dabei helfen, bessere und genauere Ziele für ihre Strategien, Aktionspläne und Programme zur Konflikt- 
verhütung festzulegen. Die EU könnte ihrerseits ihre finanzielle Unterstützung erhöhen (zum Beispiel durch ihr Instrument für Nachbarschaft, Entwicklungszusammenarbeit und internationale Zusammenarbeit), um mehrjährige OSZE-Haushalte zu ermöglichen. Dabei könnte der Schwerpunkt auf einer engeren Zusammenarbeit bei vertrauensbildenden Maßnahmen im Zusammenhang mit den langwierigen Konflikten in der OSZE-Region und der Arbeit des Hohen Kommissars für nationale Minderheiten liegen.

\section{b) Erneute Priorisierung der Rüstungskontrolle}

Die Rüstungskontrolle stand früher im Zentrum der OSZE. Hier hat sie viele Erfolge vorzuweisen. Die wachsenden Spannungen innerhalb der Organisation und die Eskalation der Gewalt bei mehreren Konflikten haben die Rüstungskontrollnormen und -mechanismen geschwächt und ausgehöhlt. Der Strukturierte Dialog über aktuelle und künftige Herausforderungen und Risiken für die Sicherheit im OSZE-Gebiet, der von den OSZE-AuBenminister*innen beim Ministerrat in Hamburg im Dezember 2016 ins Leben gerufen wurde, könnte entscheidend dazu beitragen, ein effektives Rüstungskontrollregime wiederherzustellen. Die EU hat die Möglichkeit, den Strukturierten Dialog der OSZE zu stärken, indem sie ihn in EU-Foren stattfinden lässt, wie etwa im Militärausschuss oder dem Politischen und Sicherheitspolitischen Komitee der EU. EU-Expert*innen könnten im Rahmen des Strukturierten Dialogs an infor- mellen Arbeitsgruppen und Treffen von Militärvertreter*innen auf Expertenebene teilnehmen.

\section{c) Ermöglichung von Konnektivität}

Sowohl die EU als auch die OSZE sind um Konnektivität bemüht. Innerhalb der OSZE-Region „östlich der EU“ stellt die Eurasische Wirtschaftsunion eine Alternative zum politischen und ökonomischen Integrationsprojekt der EU dar. Chinas Projekt „Neue Seidenstraße“ (Belt and Road Initiative) bietet eine weitere Vorstellung davon, wie Konnektivität aussehen kann. Im Rahmen der Zusammenarbeit zwischen der EU und der OSZE könnte die Konkurrenz zwischen den Integrationsprojekten thematisiert werden, und es könnten Grundregeln erarbeitet werden, um eine höhere Konnektivität zu ermöglichen. Die EU könnte die OSZE dabei unterstützen, eine schlüssige "Connectivity 2.0"-Agenda ${ }^{31} \mathrm{zu}$ formulieren, die einen nachhaltigen Aufschwung nach der Pandemie zum Ziel hat. Konnektivität spielt auch im Bereich Sicherheit eine immer größere Rolle. Daher haben die 27 EU- bzw. 57 OSZE-Staaten eindeutig gemeinsame Interessen, insbesondere an der Schnittstelle zwischen Klima und Sicherheit, Technik und Sicherheit sowie gute Regierungsführung und Sicherheit. Die EU könnte zudem OSZEInitiativen unterstützen, um Vertrauen in der Wirtschafts- und Umweltdimension aufzubauen, beispielsweise am Kaspischen Meer, am Schwarzen Meer und am Mittelmeer. Damit erhielte die EU Zugang zu kritischen Regionen und wür- 
de greifbare Vorteile für die Bevölkerung vor Ort schaffen. Letztlich ließe sich so der Weg ebnen für offene und konstruktive Diskussionen über Themen aus dem Bereich der „harten“ Sicherheit.

\section{d) Der Aushöhlung der Normen entgegenwirken}

Das umfassende Sicherheitskonzept der OSZE beruht auf Grundlagen, die die Teilnehmerstaaten 1975 in Helsinki und 1990 in Paris vereinbart haben. Bekräftigt wurden sie 2010 in der Gedenkerklärung von Astana. Diese Grundlagen sind im letzten Jahrzehnt zunehmend infrage gestellt worden. Daher sollte sich eine Zusammenarbeit zwischen der EU und der OSZE darauf konzentrieren, Bündnisse zwischen Staaten zu schmieden, die diese Normen verteidigen und in beiden Organisationen vertreten sind. Dabei sollte das Meinungsspektrum der 27 bzw. 57 Staaten abgebildet werden. $\mathrm{Zu}$ solchen „Freundesgruppen“ könnten OSZE-Teilnehmerstaaten gehören, die EU-Mitglieder sind, aber auch solche, die keine EUMitglieder sind. Diese Bündnisse könnten auch bei bestimmten OSZE-Institutionen angesiedelt sein, etwa beim Hohen Kommissar für nationale Minderheiten.

\section{e) Stärkung der Zusammenarbeit vor Ort}

Die EU hat in allen OSZE-Teilnehmerstaaten Delegationen, und die OSZE be- treibt aktuell in 13 Teilnehmerstaaten (allesamt „östlich der EU“) Feldoperationen. Dennoch arbeiten die EU-Delegationen und die OSZE-Feldoperationen meist nur punktuell und ad hoc zusammen. Dabei geht es meist nur um eine politische Unterstützung, was den unterfinanzierten und überlasteten OSZE-Präsenzen vor Ort nur selten längerfristig hilft. Deswegen sollte eine Priorität der EUOSZE-Kooperation darin bestehen, Verbindungen zwischen den EU-Delegationen, den EU-Sonderbeauftragten und den Botschaften der EU-Mitgliedstaaten einerseits und den OSZE-Missionen, den Sonderbeauftragten des amtierenden OSZEVorsitzenden und anderen Feldpräsenzen andererseits zu schaffen und zu festigen. Dabei könnte der Schwerpunkt auf Bildungsinitiativen liegen oder auf anderen Themen, die für die Jugend relevant sind.

\section{f) Mehr Wissensaustausch und gemeinsame Ausbildungsmöglichkeiten}

Sinnvoll wäre die Gründung eines gemeinsamen EU-OSZE-Pools von zivilen Expert"innen. Dafür sollten gemeinsame Ausbildungsveranstaltungen organisiert werden, die eine „gegenseitige Einsetzbarkeit" ermöglichen und ein Verständnis für die Kultur der jeweils anderen Organisation schaffen. Eine Initiative, die hierfür in Betracht käme, ist zum Beispiel die OSZE-Akademie in Bischkek (Kirgisistan). Sie könnte von der EU oder einzelnen Mitgliedstaaten stärker gefördert werden. Eine andere Möglichkeit bestünde darin, dass EU-Mitgliedstaaten die 
Ausbildung von OSZE-Mitarbeiter*innen für einen Einsatz durch Sachleistungen unterstützen. Vorbild hierfür könnten die Trainingskurse für die Sonderbeobachtermission in der Ukraine sein, die vom Austrian Armed Forces International Centre ${ }^{32}$ geleitet werden.

\section{g) Nutzung von Track-2- und Track-1.5- Initiativen}

Track-2- und Track-1.5-Initiativen könnten die Umsetzung der genannten Empfehlungen unterstützen. In informellen Gesprächen mit zivilgesellschaftlichen Akteuren (Track 2) oder bei Gesprächen, an denen offizielle Vertreter der Staaten und Akteure der Zivilgesellschaft beteiligt sind (Track 1.5), könnten mögliche neue Formen der Zusammenarbeit zwischen der EU und der OSZE erörtert und die Ansichten der Teilnehmerstaaten ermittelt werden. Auch die Erwartungen der 27 bzw. 57 Staaten und die roten Linien für die Nicht-EU-Staaten ließen sich so in Erfahrung bringen. Das ist entscheidend. Denn bei jeder Diskussion über eine EUOSZE-Zusammenarbeit sollte die Stärkung der OSZE im Zentrum stehen: als die wichtigste umfassende und kooperative Sicherheitsorganisation im euroatlantischen und eurasischen Raum.

\section{Endnotes}

1 Rat der EU, „Sicherheit und Verteidigung: EU schreitet auf dem Gebiet der gemeinsamen Sicherheit und Verteidigung voran“, 10. Mai 2021, https://www. consilium.europa.eu/de/press/press-releas es/2021/05/10/security-and-defence-eu-tomove-forward-on-common-security-anddefence/

2 Übersetzt nach: European External Action Service, Towards a Strategic Compass, May 2021, https://eeas.europa.eu/sit es/default/files/towards_a_strategic_com pass.pdf

3 Übersetzt nach: European Union Institute for Security Studies, Finding direction with a Strategic Compass? Reflections on the future of EU security and defence, 2021, S. 2, https://www.2021 portu gal.eu/media/5e3 pjuwv/event-report-strat egic-compass.pdf

Thomas Greminger, „Making the OSCE More Effective: Practical Recommendations from a Former Secretary General“, IFSH (ed.), OSCE Insights 1/2021, Baden-Baden: Nomos, 2022, https://doi.o $\mathrm{rg} / 10.5771 / 9783748911456-01$ https://doi. org/10.5771/9783748911456-01

5 Global Europe Program Working Group on the Future of the OSCE, „Uncommon Cause: The Future of the OSCE", in: TransAtlantic 2/2021, S. 2-4, https://ww w.wilsoncenter.org/sites/default/files/me dia/uploads/documents/Uncommon Cause - The Future of the OSCE v2.pdf

Übersetzt nach: Wolfgang Zellner, Using the OSCE More Effectively: Ideas and Recommendations, Paper for the Federal Foreign Office, 2020, S. 14, https://ifsh.de /file/publication/2021_OSZE-Studie/2021 0301_USING_THE_OSCE_MORE_EFF ECTIVELY.pdf

Auswärtiges Amt: Die Gemeinsame Sicherheits- und Verteidigungspolitik (GSVP), März 2021, https://www.auswa ertiges-amt.de/de/aussenpolitik/europa/a ussenpolitik/-/201802

Siehe z. B. Benita Ferrero-Waldner, „Die Europäische Union und die OSZE - natürliche Partner in einer vernetzten Welt “, in: OSZE-Jahrbuch 2006, BadenBaden: Nomos, 2007, S. 443-445, https://i 
fsh.de/file-CORE/documents/jahrbuch/0 6/FerrereoWaldner-dt.pdf

Übersetzt nach: European Commission, Shared vision, common action: A stronger Europe (A global strategy for the European Union's foreign and security policy), June 2016, S. 33-34, https://eea s.europa.eu/archives/docs/top_stories/pdf leugs_review_web.pdf

10 Siehe European Commission, a.a.O. (Anm. 9); European Union, The European Union's Global Strategy: Three years on, looking forward, 2019, https:/ /eeas.europa.eu/sites/eeas/files/eu_global_ strategy_2019.pdf

11 Siehe z. B. European External Action Service and European Commission, 20 deliverables for 2020: Monitoring-State of Play in February 2020, February 2020, https://www.euneighbours.eu/sites/defau lt/files/publications/2020-06/Monitoring \%20Spring\%202020_20\%20Deliverables \%20for\%202020.pdf

12 Hylke Dijkstra et al., „The EU's Partners in Crisis Response and Peacebuilding: Complementarities and Synergies with the UN and the OSCE“, in: Global Affairs 2-3/2018, 185-196; David Galbreath, „Convergence Without Cooperation? The EU and the OSCE in the Field of Peacebuilding“, in: Steven Blockmans/Jan Wouters/Tom Ruys (Hrsg.), The European Union and Peacebuilding: Policy and Legal Aspects, The Hague: Asser Press, 2010, 175-194.

13 Alyson J. K. Bailes/Jean-Yves Haine/ Zdzislaw Lachowski, „Überlegungen zur Beziehung von OSZE und EU“, in: IFSH (Hrsg.), OSZE-Jahrbuch 2007, Baden-Baden: Nomos, 2008, 69-83, S. 82, https://ifs h.de/file-CORE/documents/yearbook/eng lish/07/BailesHaineLachowski-en.pdf

14 Siehe auch Deutscher Bundestag, Antrag der Fraktionen CDU/CSU, SPD, FDP und BÜNDNIS 90/DIE GRÜNEN: 45 Jahre Schlussakte von Helsinki, 30 Jahre Charta von Paris - Die Organisation für
Sicherheit und Zusammenarbeit in Europa für künftige Aufgaben stärken, Deutscher Bundestag Drucksache 19/19140, 2020, https://dip21.bundestag.de/dip21 /btd/19/135/1913551.pdf

15 Siehe OSCE, Istanbul Document 1999 (Charter for European Security and Istanbul Summit Declaration), PCOEW389, 1999, S. 43-45, https://www.osce.org/mc/ 39569

16 Übersetzt nach: Council of the European Union, Draft Council Conclusions on EU-OSCE Cooperation in Conflict Prevention, Crisis Management and Post-Conflict Rehabilitation, Doc. No. 14527/1/03, 10 November 2003, https:// data.consilium.europa.eu/doc/document/ ST-14527-2003-REV-1/en/pdf

17 Council of the European Union, a.a.O. (Anm. 16), S. 2.

18 OSZE, Geschäftsordnung der Organisation für Sicherheit und Zusammenarbeit in Europa, MC.DOC/1/06, 1. November 2006, S. 8 und S. 10, https://www.osce.or g/files/f/documents/4/9/22777.pdf

19 European External Action Service, First EU-OSCE high-level meeting takes place in Brussels, 12. Dezember 2018, https://e eas.europa.eu/headquarters/headquartershomepage/55370/first-eu-osce-high-levelmeeting-takes-place-brussels_en; siehe auch OSCE, Strengthening OSCE-EU Co-Operation Discussed at First Annual High-Level Meeting Held in Brussels, 13 December 2018, https://www.osce.org/se cretary-general/406682

20 Siehe Delegation of the European Union to the International Organisations in $\mathrm{Vi}$ enna, Organisation for Security \& Co-operation in Europe (OSCE), Juni 2016, https://eeas.europa.eu/delegations/vien na-international-organisations/2297/orga nisation-security-co-operation-europe-osc e

21 Siehe European Council/Council of the European Union, Working Party on the OSCE and the Council of Europe, https:/ 
/www.consilium.europa.eu/en/council-eu /preparatory-bodies/working-party-osce-a nd-council-of-europe/

22 Michael F. Harsch, The Power of Dependence: NATO-UN Cooperation in Crisis Management, Oxford: Oxford University Press, 2015.

23 European Commission, „The EU provides further satellite imagery support to the OSCE Special Monitoring Mission in Ukraine“, 24 March 2017, https://ec.euro pa.eu/commission/presscorner/detail/es/I P 17 729

24 Rafael Biermann/Joachim A. Koops, „Studying Relations Among International Organizations in World Politics: Core Concepts and Challenges“, in: Rafael Biermann/Joachim A. Koops (Hrsg.), The Palgrave Handbook of Inter-Organization Relations, London: Palgrave MacMillan, 2017, S. 22.

25 Darin nicht eingeschlossen sind die operativen Kosten für die OSZE-Sonderbeobachtermission in der Ukraine.

26 Eine weitere Komplikation für die $\mathrm{Zu}$ sammenarbeit zwischen der EU und der OSZE, vor allem in der politisch-militärischen Dimension, beruht auf der Tatsache, dass 26 der EU-Mitgliedstaaten auch NATO-Mitglieder sind, deren 30 Mitglieder wiederum allesamt der OSZE angehören.

27 Philip Remler, The OSCE as Sisyphus: Mediation, Peace Operations, Human rights, IAI Papers 21/16, Istituto Affari Internazionali, April 2021, https://www.i ai.it/sites/default/files/iaip2116.pdf

28 Barbara Gray, „Intervening to Improve Inter-organizational Relationships", in: Steve Cropper/Mark Ebers/Chris Huxham/Peter Smith Ring (Hrsg.), The Oxford Handbook of Inter-organizational Relations, Oxford: Oxford University Press, 2008, 664-690.
29 Siehe Dijkstra et al., a.a.O. (Anm. 12) und Galbreath, a.a.O. (Anm. 12).

30 Dies ist besonders wichtig angesichts des Beschlusses des Europäischen Rats vom Juni 2021, die EU-Russland-Gipfel nicht fortzuführen - entgegen dem gemeinsamen Vorschlag Frankreichs und Deutschlands, die Gipfel weiterhin stattfinden zu lassen, um den Beziehungen zwischen der EU und Russland eine ähnliche Basis $\mathrm{zu}$ verschaffen wie den US-Russland-Beziehungen, wie es beim Treffen zwischen Wladimir Putin und Joe Biden am 17. Juni 2021 in Genf vereinbart wurde. Die Möglichkeiten, die die OSZE als Forum bietet, können zwar keinen direkten Dialog zwischen der EU und Russland ersetzen. Doch die bilateralen Beziehungen zwischen der EU und Russland sollten nicht die Beziehungen zu Russland innerhalb des multilateralen Kontexts der OSZE bestimmen. Die OSZE bietet eine der wenigen verbliebenen Möglichkeiten für einen institutionalisierten Dia$\log$, in den Russland, die EU und all ihre Mitgliedstaaten eingebunden sind.

31 Siehe Stefan Wolff, „China: A Challenge or an Opportunity for the OSCE?", Security and Human Rights Monitor, 22 April 2021, https://www.shrmonitor.org/ china-a-challenge-or-an-opportunity-for-t he-osce-shrm/

32 Das Programm wurde 2014 ins Leben gerufen und hat bislang rund $1.000 \mathrm{Be}$ obachter*innen ausgebildet. Siehe OSCE, „OSCE Secretary General, high-level OSCE officials mark fifth anniversary of AUTINT-run pre-deployment training for Special Monitoring Mission to Ukraine in Götzendorf, Lower Austria“, OSCE, 12 June 2019, https:/www.osce.or $\mathrm{g} /$ secretary-general/422819 